\title{
Investigation of tellurium-implanted silicon*
}

\author{
T. F. Lee ${ }^{\dagger}$, R. D. Pashley $y^{\ddagger}$, T. C. McGills, and J. W. Mayer \\ California Institute of Technology, Pasadena, California 91109 \\ (Received 16 August 1974)
}

Hall and sheet-resistivity measurements as a function of temperature combined with layer removal have been used to study Si implanted with Te at energies up to $220 \mathrm{keV}$. At low doses $\left(\lesssim 4 \times 10^{12}\right.$ $\mathrm{cm}^{-2}$ ), Te has a donor level with a $140-\mathrm{meV}$ activation energy. The activation energy decreases at higher Te doses and is approximately equal to zero for Te doses $\gtrsim 10^{15} \mathrm{~cm}^{-2}$. At high dose levels, the number of conduction electrons per unit area $N_{s}$ is more than an order of magnitude below the number of $\mathrm{Te}$ per unit area. High-temperature anneal treatments followed by quenching did not produce a substantial increase in $N_{s}$, suggesting that the formation of Te clusters was not responsible for the low value of $N_{s}$. Also, channeling measurements indicated a high substitutional fraction. Based on differential Hall measurements on samples implanted with phosphorus, with and without $\mathrm{Si}$ predamage, we conclude that residual radiation damage is not a major factor. A theoretical calculation, which includes the effect of decrease of activation energy with increasing impurity concentrations, indicated that the number of conduction electrons could be much less than the number of implanted $\mathrm{Te}$ even though the apparent activation energy is almost zero. Although the results of theoretical calculation do not give quantitative agreement with the experimental results, they do confirm the changes in apparent activation energy with concentration.

\section{INTRODUCTION}

Generally, deep-level impurities have low solid solubilities in semiconductors. Therefore, by conventional doping techniques such as thermal diffusion, it is difficult to heavily dope semiconductors with deep-level impurities. Ion implantation, ${ }^{1}$ however, offers the possibility for introducing high concentrations of deep-level dopants in silicon. MeV He channeling studies have been made of the lattice location of a wide variety of dopant species in $\mathrm{Si} .{ }^{2}$ Of these dopant species, tellurium would appear to be attractive for the evaluation of electrical properties because of its high substitutional fraction ${ }^{3}$ and deep level. ${ }^{4}$

When Te is implanted at low doses $\left(\leqslant 4 \times 10^{12} \mathrm{~cm}^{-2}\right)$, it acts as an $n$-type dopant with an activation energy of $140 \mathrm{meV} .{ }^{5}$ However, samples implanted with high doses (typically $10^{14}-10^{15} \mathrm{~cm}^{-2}$ ) exhibit a somewhat puzzling behavior; the number of conduction electrons has a temperature activation energy which is about zero; and the measured number of conduction electrons is more than an order of magnitude smaller than the number of implanted Te. ${ }^{6}$ From conventional semiconductor theory, ${ }^{7}$ one would anticipate that for heavily doped semiconductors which have a zero activation energy, the number of conduction electrons would be nearly equal to the number of substitutional impurities. In this work, we pursue the apparent contradiction between a zero activation energy and the small number of conduction electrons as compared to the number of substitutional Te dopants.

We have investigated several experimental aspects that could influence the measured electrical activity: (i) the nonuniform depth distribution of the implanted Te, (ii) the influence of radiation damage, and (iii) formation of substitutional clusters of Te. Previous Hall-effect measurements on high-dose Te-implanted samples ${ }^{6}$ were made without use of layer-removal techniques. ${ }^{8,9}$ The measured number of conduction electrons $/ \mathrm{cm}^{2}, N_{s}$, is usually smaller than the actual number present due to the mobility weighting factor. This weighting effect can be overcome by differential measurements which combine Hall-effect and resistivity measurements with layerremoval techniques. This differential technique coupled with measurements as a function of temperature has been found to give activation energies consistent with those for bulk doped samples for silicon implanted with indium and tellurium at low concentrations. ${ }^{5,10}$ In this work we have used differential techniques to obtain the temperature dependence of the carrier concentration for substitutional Te concentrations between $10^{17}$ and $10^{20} / \mathrm{cm}^{3}$.

For implanted samples, residual radiation damage can remain after high-temperature anneal. The radiation defects may act as compensation centers and thus reduce the number of conduction electrons. To investigate the effect of implantation radiation damage, we performed electrical measurements on low-dose $\left(10^{12}-10^{13} / \mathrm{cm}^{2}\right)$ phosphorus-implanted Si samples with and without predamage introduced by a higher dose $\left(10^{15} / \mathrm{cm}^{2}\right)$ of implanted $\mathrm{Si}$ ions. If the radiation damage is responsible for the reduction in the number of electrons $/ \mathrm{cm}^{2}$ for the Te case, we would expect that the electrical activity of phosphorus-implanted samples with predamage would be much less than the electrical activity of phosphorusimplanted samples without predamage.

In arsenic-diffused samples, it has been found ${ }^{11}$ that the electrical activity is reduced by long heat treatment at temperatures of $500-970^{\circ} \mathrm{C}$. The electrical activity could be increased by heating at higher temperatures (approximately $1100^{\circ} \mathrm{C}$ ) and then quenching. The reduction of electrical activity was attributed to the formation of As clusters which could be dissociated during high-temperature processing. Similar effects may play a role in high-dose Te-implanted samples where the concentration of $\mathrm{Te}\left(10^{19}-10^{20} \mathrm{~cm}^{-3}\right)$ can be orders of magnitude above the reported ${ }^{4}$ thermal equilibrium solid solubility of $10^{17} \mathrm{~cm}^{-3}$. To investigate the influence of cluster formation we employed high-temperature heat treatment followed by quenching for some samples. In analogy with As results, this treatment should lead to 


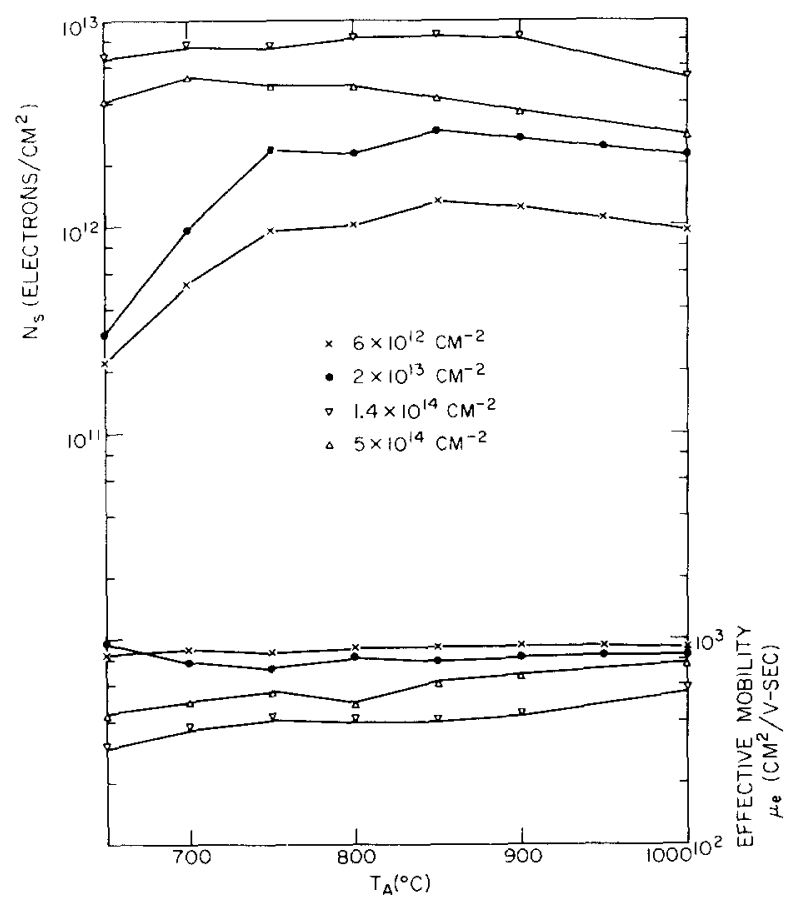

FIG. 1. Anneal behavior of the surface electron concentration $N_{s}$ and the effective mobility $\mu_{e}$ for silicon implanted at room temperature with $220-\mathrm{keV}$ Te ions.

high electrical activity if formation of Te clusters were responsible for the relatively low electron concentrations.

From a theoretical approach, we used the model proposed by Lee and McGill ${ }^{12}$ to treat the decrease of activation energy with increasing impurity concentrations. In this case, we calculated the conduction electron concentrations as a function of temperature for different $\mathrm{Te}$ concentrations to determine whether there is a contradiction between the almost zero activation energy and the reduction in conduction electron concentrations.

This paper is organized in the following way. Section II contains the experiment and analysis procedures. In Sec. III we describe the procedure of calculating electron concentrations as a function of temperature. In Sec. IV, we present the experimental results and compare them with theoretical calculations.

\section{EXPERIMENTAL AND ANALYSIS PROCEDURES}

\section{A. Sample preparation}

Implantations of tellurium were made at energies between 100 and $220 \mathrm{keV}$ in etch-polished slices of floatzoned 10 - and $2000-\Omega \mathrm{cm} p$-type silicon. The projected ranges for 100 - and $220-\mathrm{keV}$ tellurium implantation in silicon are 456 and $870 \AA$, respectively. ${ }^{13}$ Ion doses ranged between $4 \times 10^{12}$ and $1.4 \times 10^{15} \mathrm{~cm}^{-2}$. Ion implantations of phosphorus were made at energies between 7 and $190 \mathrm{keV}$ and ion doses between $3 \times 10^{12}$ and $3 \times 10^{13} \mathrm{~cm}^{-2}$. The projected range of silicon implantation for predamage was deeper than those of phosphorus implantations; the silicon dose was $10^{15} \mathrm{~cm}^{-2}$. All the substrates were at room temperature.

Hall-effect and sheet resistivity measurements were made using the van der Pauw configuration. ${ }^{14}$ The van der Pauw pattern was defined on the implanted surface by masking with photoresist followed by mesa etching. The mesa structure in combination with the $p-n$ junction between the implanted layer and substrate isolated the region of interest.

After the van der Pauw patterns were put on the implanted specimens, they were placed in a quartz tube furnace with flowing $\mathrm{N}_{2}$ to anneal out the radiation damage. The P-implanted samples were annealed to $850^{\circ} \mathrm{C}$ for $15-30 \mathrm{~min}$.

For Te-implanted samples, isothermal anneal cycles have been performed to determine the anneal temperature where the number of electrons $/ \mathrm{cm}^{2}$ was near the maximum value. The anneal behavior of several samples were shown in Fig. 1, in which the measured number of electrons $/ \mathrm{cm}^{2}$ and the electron mobility were plotted as a function of anneal temperature. As found previously, ${ }^{15}$ the number of electrons $/ \mathrm{cm}^{2}$ is much lower than the number of $\mathrm{Te} / \mathrm{cm}^{2}$. For the highest dose implant shown there is a factor of 60 difference between the two values. As shown in Fig. 1, the number of electrons $/ \mathrm{cm}^{2}$ and the electron mobility stayed essentially at the same value over the anneal temperature between 800 and $1000^{\circ} \mathrm{C}$. Therefore, anneal temperatures between 800 and $1000^{\circ} \mathrm{C}$ for $15-30 \mathrm{~min}$ have been chosen for all the samples.

MeV He ion backscattering and channeling measurements were made on several annealed Te-implanted samples to confirm the previous results which indicated a high $(60 \%)$ substitutional percent of Te atoms. ${ }^{3}$ The highest substitutional percent we obtained was $80 \%$ for a sample implanted with $1.4 \times 10^{14} \mathrm{Te} / \mathrm{cm}^{2}$ and the lowest was $30 \%$ for a dose of $1.4 \times 10^{15} \mathrm{Te} / \mathrm{cm}^{2}$. As pointed out previously, these results indicate that the low electrical activity of Te-implanted layers cannot be due entirely to nonsubstitutional Te.

\section{B. Layer-removal techniques}

Layer removal was accomplished by using oxide layerstripping techniques. In practice, a layer of silicon dioxide was anodically grown on the sample and then removed by etching. ${ }^{6} \mathrm{~A}$ vinyl mask was used to define the area of the anodic oxide on the sample which was then securely clamped below a hole at the bottom of a Teflon beaker. The anodic solution contained in the beaker was made from $97 \% \mathrm{~N}$-methylacetamide, $2 \%$ triply distilled water, and $1 \%$ potassium nitrate by weight. A constant current density of $9 \mathrm{~mA} / \mathrm{cm}^{2}$ in the presence of high-intensity light was used during the process of anodization.

Ellipsometry measurements were made to determine the thickness-voltage dependence. The oxide thicknesses were found to be reproducible within $5 \%$. The oxide layer was stripped with concentrated hydrofluoric acid (HF). The thickness of the removed silicon layer was assumed to be $43 \%$ of the oxide layer thickness. 


\section{Electrical measurements}

Hall effect and sheet resistivity were measured as a function of temperature. The desired temperature was maintained through heat exchange in a gas-flow liquidnitrogen cryostat. The platinum sensor and controller held temperatures to within $\pm 0.2^{\circ} \mathrm{K}$. A magnetic field of $4 \mathrm{kG}$ has been employed for Hall-effect measurements Measurements were performed using pressure contact to the implanted layers.

As a result of Hall-effect and sheet-resistivity measurements, the number of electrical carriers $/ \mathrm{cm}^{2}, N_{s}$, and sheet resistivity $\rho_{s}$ can be obtained if we assume that the Hall mobility is equal to drift mobility. This assumption is valid for heavily doped semiconductor samples. ${ }^{16}$ For low-dose samples, this assumption may give rise to, at most, a factor-of-2 error. By combining the layer-removal technique with Hall-effect and sheet-resistivity measurements we can obtain the average carrier concentration and resistivity of the removed thin layer through the relationship ${ }^{8}$

$$
\begin{gathered}
n_{i}=\left[\left(\frac{1}{\rho_{s}}\right)_{i}-\left(\frac{1}{\rho_{s}}\right)_{i+1}\right]^{2}\left\{d_{i}\left[\left(\frac{1}{\rho_{s}^{2} N_{s}}\right)_{i}-\left(\frac{1}{\rho_{s} N_{s}}\right)_{i+1}\right]\right\}^{-1}, \\
\rho_{i}=d_{i}\left[\left(\frac{1}{\rho_{s}}\right)_{i}-\left(\frac{1}{\rho_{s}}\right)_{i+1}\right]^{-1},
\end{gathered}
$$

where $\left(N_{s}\right)_{i+1}$ and $\left(\rho_{s}\right)_{i+1}$ are measured carriers $/ \mathrm{cm}^{2}$ and sheet resistivity after the removal of the $i$ th layer; $d_{i}$ is the thickness of the removed $i$ th layer.

\section{Quenching}

To perform the quenching experiments, we set the furnace at an upright position so that the samples could move freely in the vertical direction. The samples were placed in a quartz basket with several holes at the bottom. At the end of the annealing time period, the quartz basket and samples were dropped within $0.2 \mathrm{sec}$ into a beaker containing deionized water. The samples were cycled through a series of heat treatments which involved anneal at $1000^{\circ} \mathrm{C}$ followed by a quench and then anneal at $850^{\circ} \mathrm{C}$ without a quench. Electrical measurements were made before and after each anneal.

\section{THEORY OF ACTIVATION ENERGY VARIATION}

The ratio of conduction electron concentration to the impurity concentration at various temperatures depends upon the impurity activation energy. Hence, any variation in the impurity activation energy would cause a change in the values of the electron-to-impurity-concentration ratio. It has been reported that the impurityto-band activation energy decreases at high impurity concentrations in semiconductors. ${ }^{17}$ Recently, Lee and McGill $^{12}$ have worked out a theory for the variation of activation energy with impurity concentrations. They pointed out that the donor activation energy decreases because of both donor-level shifting and donor-level broadening.

To illustrate the model of their theory, the conduction band density of states $N_{c}(E)$ and the donor-level density of states $\rho_{i}(E)$ are plotted in Fig. 2 for the cases of

both low donor concentrations and high donor concentrations. For the low donor concentration case, the conduction band edge and the discrete donor level are well defined. The donor activation energy is unique and equal to $E_{D}$. For the high donor concentration case, in addition to the strict shift of conduction band edge toward the donor level $\Delta E_{c}$, the broadening of the donor states and the tail of the conduction band edge also contribute to the decrease of donor activation energy. Due to the donor-state broadening effect, some impurity states shift upward toward the conduction band edge and some shift downward away from conduction band edge, as shown in Fig. 2. The upward shifted states contribute partly to the decrease of the activation energy and the downward shifted states are mostly occupied by electrons and thus correspond to the nonionized portion of Te impurities. The donor activation energy is not uniquely defined and thus varies as a function of temperature. Further, even though the apparent activation can decrease to values near zero, the donor atoms are not all ionized.

Using this physical model, we calculated the conduction electron concentration as follows: The conduction electron concentration $n$ is given by the standard expression

$$
n=\int_{-\infty}^{\infty} \frac{N_{c}\left(E-\Delta E_{c}\right)}{1+\exp \left[\left(E-E_{f}\right) / K_{B} T\right]} d E
$$

where $E_{f}$ is Fermi energy, $K_{B}$ is the Boltzmann constant, $T$ is absolute temperature, $N_{c}(E)$ is conductionband density of states, and $\Delta E_{c}$ is the lowering of the conduction band edge due to attraction between conduction electrons and ionized donors. Because of conduction band edge smearing due to potential fluctuation, the

Density of States for

Low Donor Concentrations

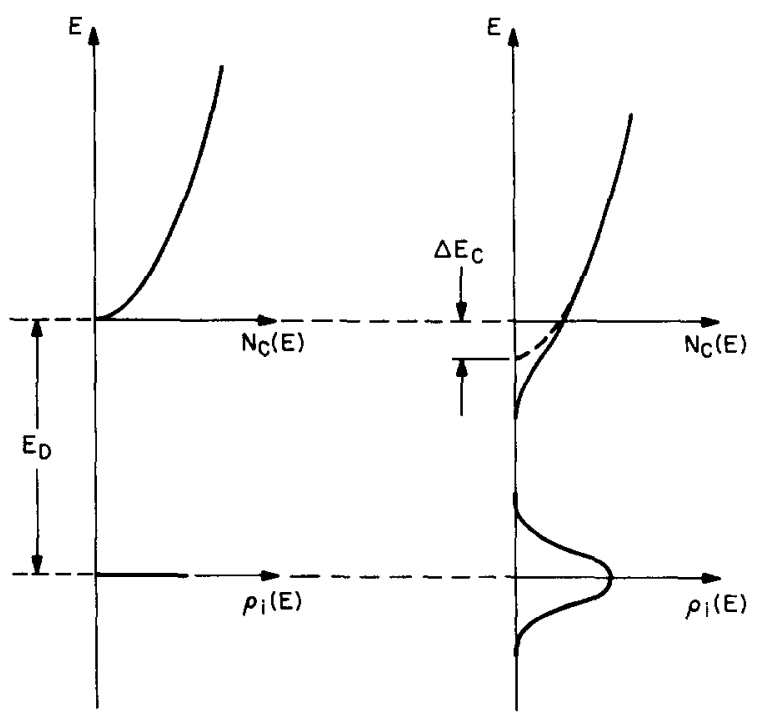

FIG. 2. The physical model for the decrease of activation energy with increasing impurity concentrations. 


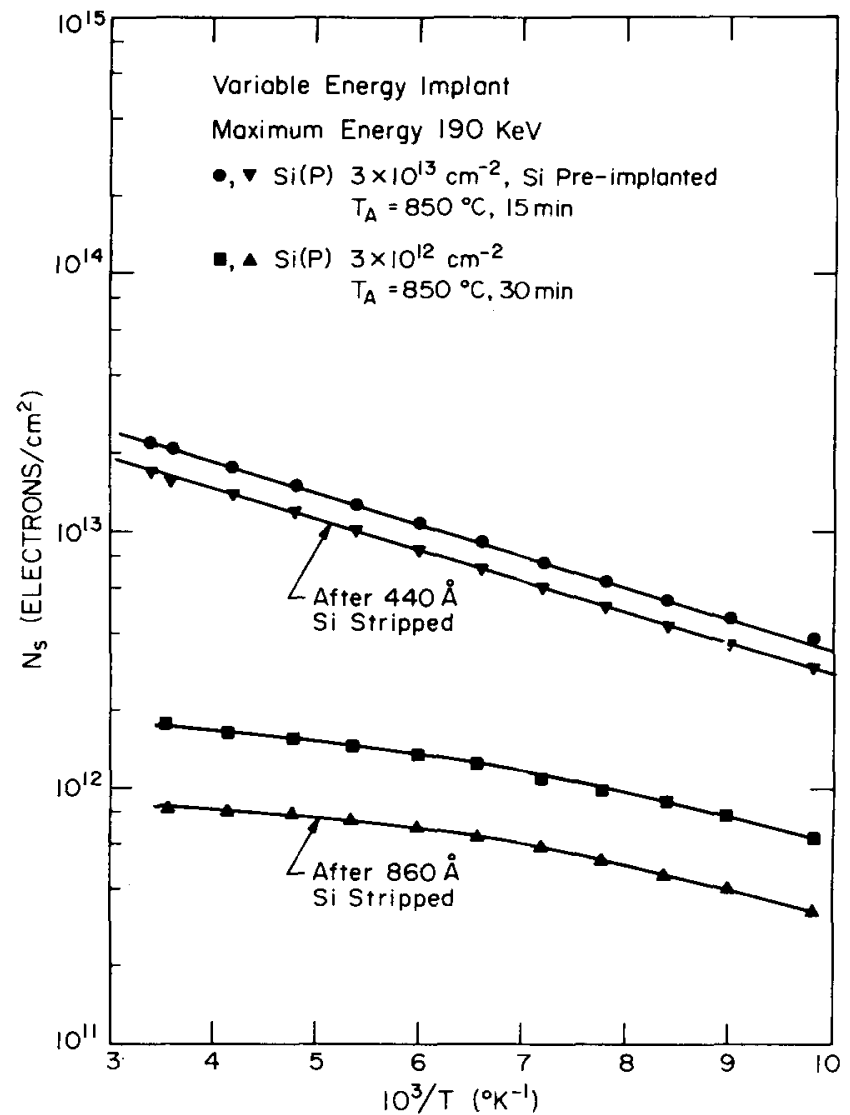

FIG. 3. The surface electron concentration $N_{s}$ vs reciprocal temperature for two $\mathrm{P}$-implanted silicon samples before and after a thin layer was removed.

shape of $N_{c}(E)$ is different from that of intrinsic Si. The expressions for $N_{c}(E)$ and $\Delta E_{c}$ are given in the Appendix. Similarly, the concentration of ionized donors is defined as

$$
N_{D}^{+}=\int_{-\infty}^{\infty} \frac{\rho_{i}\left(E-E_{D}\right)}{1+g \exp \left[\left(E_{f}-E\right) / K_{B} T\right]} d E,
$$

where $g$ is the degeneracy factor for the donor ground state, $\rho_{1}(E)$ is the donor-level density of states, and $\left(-E_{D}\right)$ is the donor ionization energy at low donor concentrations. The factor $\rho_{i}(E)$ depends upon the effects of donor-level broadening due to donor-electron wavefunction overlapping and the effect of donor-state spreading due to potential fluctuation. The expression for $\rho_{i}(E)$ is also given in the Appendix. For donors, such as $\mathrm{P}$ in $\mathrm{Si}$, the hydrogen model is suitable to describe the donor states and thus $g$ is assumed to be equal to 2. For donors, such as $\mathrm{Te}$ in $\mathrm{Si}$, the helium model seems to be appropriate to describe the donor states and thus $g$ is assumed to be $\frac{1}{2} .{ }^{18,19}$

With the presence of compensating acceptor concentration $N_{A}$, charge neutrality leads to

$$
n+N_{A}=N_{D}^{+} \text {, }
$$

which determines the Fermi level $E_{f}$ and, in turn, determines the conduction electron concentration $n$ from Eq. (3.1). The presence of compensation centers not only reduces the number of electrons but also introduces additional broadening due to increased potential fluctuations. ${ }^{12}$

\section{RESULTS}

High-temperature anneal treatment after ion implantation may still leave some residual damage. The residual damage may act like compensation centers and thus reduce the number of electrons $/ \mathrm{cm}^{2}$. For the purpose of investigating the influence of residue radiation damage on the electrical properties of ion-implanted samples, we investigated the influence of damage caused by preimplantation of $\mathrm{Si}$ ions.

\section{A. P-implanted Si samples}

In Fig. 3 the measured number of electrons $/ \mathrm{cm}^{2}, N_{s}$, vs $1 / T$ are given for two samples; one of the sample was phosphorus implanted to $3 \times 10^{13} \mathrm{~cm}^{-2}$ with $S i$ ion predamage and the other sample was phosphorus implanted to $3 \times 10^{12} \mathrm{~cm}^{-2}$ without predamage. For both of the samples, the numbers of electrons $/ \mathrm{cm}^{2}$ at room temperature were equal to two-thirds of phosphorus concentrations. Therefore, the Si ion predamage does not seem to result into significant reduction of number of conduction electrons at room temperature.

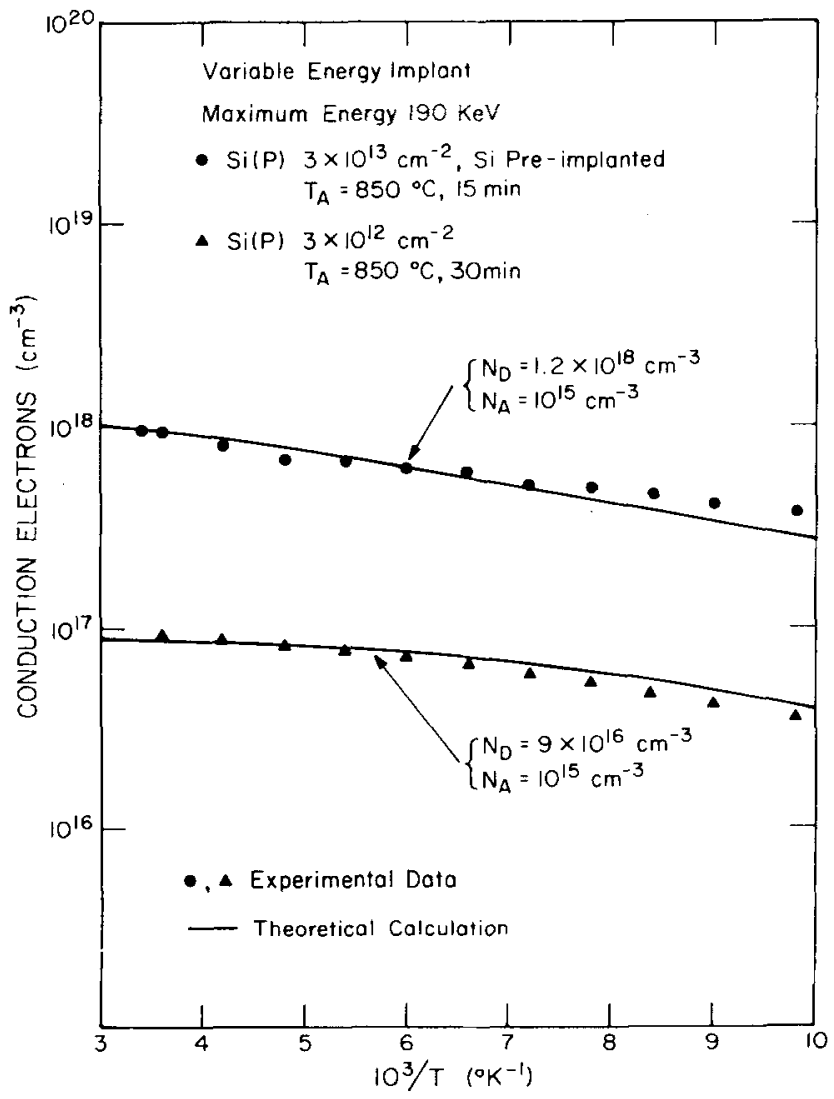

FIG. 4. The conduction electron concentrations of differential measurements vs reciprocal temperature for $\mathrm{P}$-implanted silicon samples. The calculated conduction electron concentrations vs reciprocal temperature for $\mathbf{P}$ in silicon are shown for comparison. 


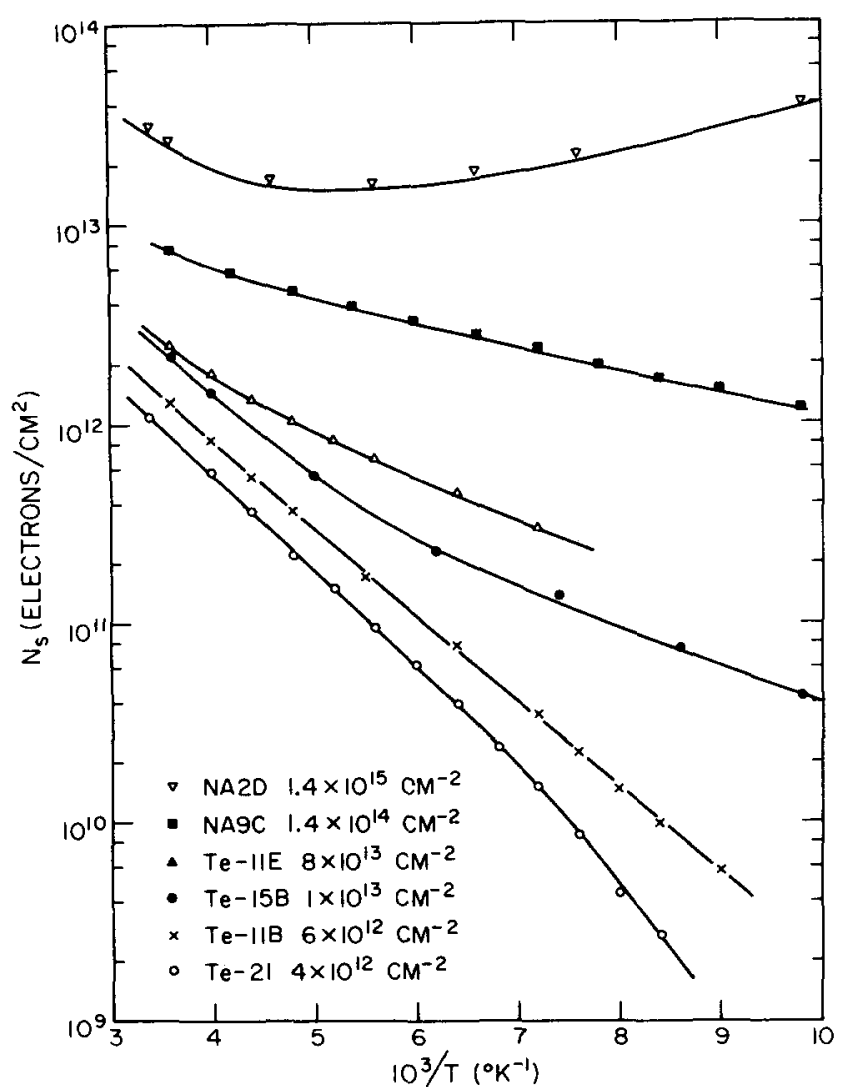

FIG. 5. The surface electron concentration $N_{s}$ vs reciprocal temperature for Te-implanted silicon silicon samples.

To investigate the effect of residual radiation damage in more detail, we used differential measurements to obtain the carrier concentration as a function of temperature. Therefore, in Fig. 3 the measured numbers of electrons $/ \mathrm{cm}^{2}$ vs $1 / T$ are shown for both samples after a layer was removed. The results of the differential measurements are shown in Fig. 4 which gives the concentration of electrons $/ \mathrm{cm}^{3}$ vs $1 / T$. The conduction electron concentrations at room temperature are about $10^{18} \mathrm{~cm}^{-3}$ for the sample implanted with phosphorus $3 \times 10^{13} \mathrm{~cm}^{-2}$ and about $10^{17} \mathrm{~cm}^{-3}$ for the sample implanted with phosphorus $3 \times 10^{12} \mathrm{~cm}^{-2}$. To compare the experimental results with theoretical calculations (see Appendix) we also plotted in Fig. 4 the calculated conduction electron concentrations as a function of $1 / T$. The donor concentrations $N_{D}$ and the compensating acceptor concentrations $N_{A}$ were chosen to give best agreement between theory and experiment. The numerical calculations with $N_{D}=1.2 \times 10^{18} \mathrm{~cm}^{-3}$ and $N_{A}=10^{15} \mathrm{~cm}^{-3}$ was in good agreement with the experimental results of the sample implanted with phosphorus $3 \times 10^{13} \mathrm{~cm}^{-2}$. Similar ly, the numerical calculation with $N_{D}=9 \times 10^{16} \mathrm{~cm}^{-3}$ and $N_{A}=10^{15} \mathrm{~cm}^{-3}$ was also in satisfactory agreement with the experimental results of the sample implanted with phosphorus $3 \times 10^{12} \mathrm{~cm}^{-2}$. The concentrations of compensating centers $N_{A}$ are two to three orders of magnitude below the phosphorus concentration. The good agreement between the theory and experiment on phosphorus-implanted Si samples indicated that the effect of the residual radiation damage was negligible. Although this is not conclusive evidence for an absence of dam- age effects in the $\mathrm{Te}$ case, it does suggest that radiation damage is not the major factor responsible for the low activity.

\section{B. Te-implanted Si samples}

We investigated the electrical properties of $\mathrm{Te}$-implanted samples by performing Hall-effect and resistivity measurements. From the sign of the Hall voltage, we deduced that implanted $\mathrm{Te}$ has donor behavior in $\mathrm{Si}$, in agreement with the result of $\mathrm{Te}$-doped bulk Si samples. ${ }^{4}$

The anneal of Te-implanted samples to $1000^{\circ} \mathrm{C}$ and rapid quench to room temperature did not give significant increase of conduction electrons $/ \mathrm{cm}^{2}$. As discussed in Sec. II D, samples were processed through several quench and no-quench cycles. The values of $N_{s}$ changed by about $10 \%$ throughout the quenching steps, but no systematic trend was noted. Therefore, we do not believe that the formation of Te substitutional clusters could account for the entire difference between the number of electrons $/ \mathrm{cm}^{2}$ and the number of implanted $\mathrm{Te}$ atoms. It is possible, of course, that a temperature of $1000^{\circ} \mathrm{C}$ is not sufficient to dissociate Te clusters or that faster quenching is required.

The measured numbers of conduction electrons $/ \mathrm{cm}^{2}$, $N_{s}$, vs $1 / T$ for several Te-implanted samples were plotted in Fig. 5. For these samples, the lowest dose is $4 \times 10^{12} \mathrm{~cm}^{-2}$ and the highest dose is $1.4 \times 10^{15} \mathrm{~cm}^{-2}$. In Fig. 5 , the slopes of $\log N_{\text {s }}$ vs $1 / T$ decrease as the $\mathrm{Te}$ concentrations increase. This indicates qualitatively that the $\mathrm{Te}$ activation energy decreases as $\mathrm{Te}$ concentration increases. For the sample implanted with $1.4 \times 10^{15} \mathrm{Te} \mathrm{cm}^{-2}$, the activation energy is approximately equal to zero but the number of conduction electrons/ $\mathrm{cm}^{2}$ is about $2 \times 10^{13} \mathrm{~cm}^{-2}$ which is almost two orders of magnitude less than the number of implanted Te atoms. The upward curvature in the data for the high-dose sample caused by mobility weighting has been observed in other measurements on Te-implanted samples. ${ }^{6}$

The results of the differential measurements for three of the samples are plotted vs $1 / T$ in Fig. 6 . The slope of the curve for the sample implanted with $4 \times 10^{12} \mathrm{Te} \mathrm{cm}^{-2}$ yields an activation energy of $140 \mathrm{meV}$, which agrees with the result of Te-doped bulk Si samples. ${ }^{4}$ As also found in Fig. 5 , the activation energy in Fig. 6 decreases with increasing Te concentration. For the sample implanted with $1.4 \times 10^{15} \mathrm{Te} / \mathrm{cm}^{2}$, the measured conduction electron concentration does not vary appreciably between room temperature and $100{ }^{\circ} \mathrm{K}$.

To compare the experimental results with the results of the theoretical calculations, we also plotted in Fig. 6 the calculated conduction electron concentrations vs 1 / $T$ for different $T e$ concentrations. The Te concentrations $N_{D}$ and the compensating acceptor concentrations $N_{A}$ shown in Fig. 6 were chosen to give best agreement with the experimental results. The numerical calculation with $N_{D}=3 \times 10^{19} \mathrm{~cm}^{-3}$ and $N_{A}=2.5 \times 10^{18} \mathrm{~cm}^{-3}$ indicated that the conduction electron concentrations varied very little over the temperature range between room temperature and $100^{\circ} \mathrm{K}$. As we can see from Fig. 6 the experimental and the theoretical results have some 


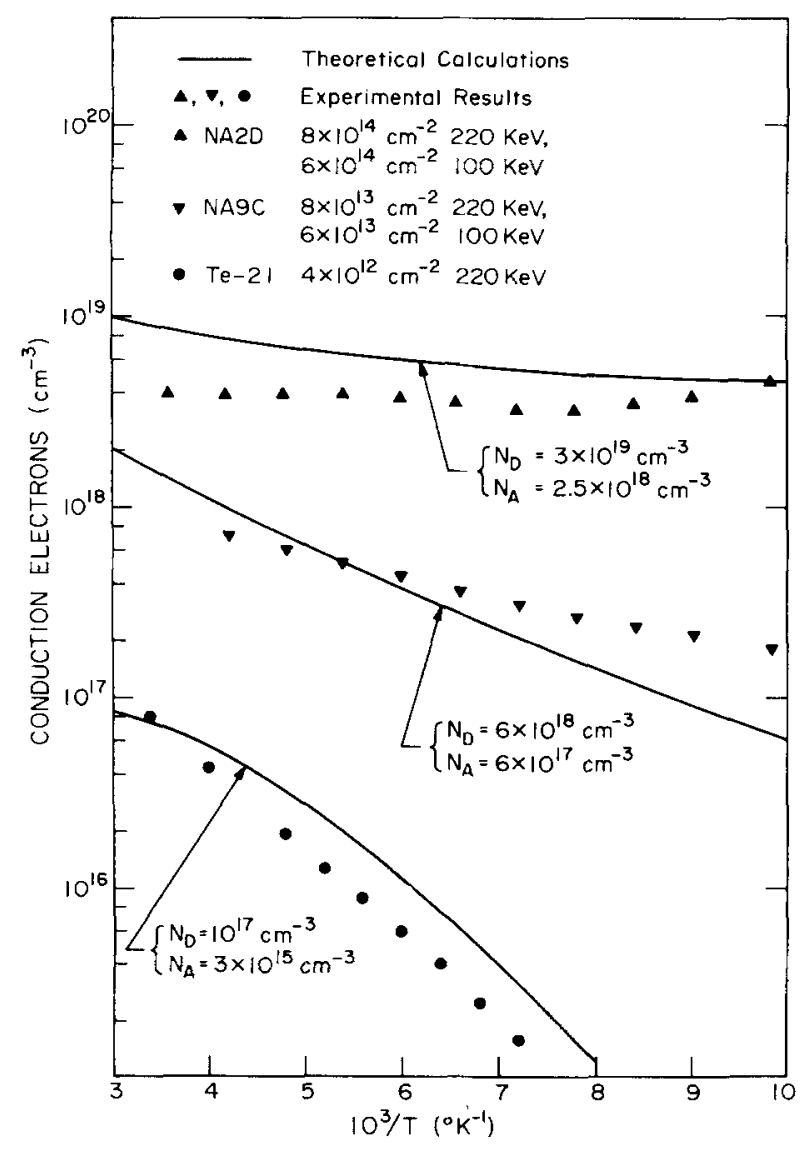

FIG. 6. The conduction electron concentrations of differential measurements vs reciprocal temperature for Te-implanted silicon samples. The calculated conduction electron concentrations vs reciprocal temperature for $\mathrm{Te}$ in silicon are shown for comparison.

similar behavior. First, both the theoretical and the experimental results have the same trend in that the activation energy decreases with increasing $\mathrm{Te}$ concentrations, as we expected. Second, for high $\mathrm{Te}$ concentrations (greater than $10^{19} \mathrm{~cm}^{-3}$ ), the activation energy is almost equal to zero but the conduction electron concentrations are much smaller than the Te concentrations. On a quantitative basis there is still a discrepancy between the calculated and measured number of electrons per unit volume. The origin of this discrepancy is not known at present.

\section{CONCLUSION}

For high-dose Te-implanted Si samples, the number of electrons $/ \mathrm{cm}^{2}$ are found to be much less than the implanted number of $\mathrm{Te} / \mathrm{cm}^{2}$, while the activation energy was approximately equal to zero. By use of the layerremoval technique in combination with Hall-effect and resistivity measurements, we were able to investigate the electrical properties of Te-implanted Si samples in more detail. The effect of the residual radiation damage has been investigated through the simulation of radiation damage by $\mathrm{Si}$ ion predamage in phosphorus-implanted samples. The negligible influence of the residual radiation damage on the electrical properties of phosphorusimplanted samples, with or without Si ion predamage, led us to assume that the residual radiation damage did not substantially reduce the electron concentrations of the Te-implanted Si samples. Quenching of the Te-implanted samples after a $1000^{\circ} \mathrm{C}$ anneal has been found to give little increase of number of conduction electrons $/ \mathrm{cm}^{2}$. Therefore, we do not believe that the formation of inactive substitutional Te clusters is entirely responsible for the much smaller number of conduction electrons $/ \mathrm{cm}^{2}$ as compared to the implanted $\mathrm{Te}$ atoms.

Theoretical calculations which included the effects of decrease of activation energy with increasing impurity concentrations gave qualitative agreement with the experiment results. Both the theoretical and experimental results indicated that the activation energy decreases approximately to zero for Te concentrations higher than $10^{19} \mathrm{~cm}^{-3}$. Further, not all the Te are ionized even though the activation energy is almost zero. This is understandable from our model of the variation of activation energy with impurity concentrations. In addition to the strictly downward shifting of the conduction band edge, the broadening of impurity states and the smearing of the conduction band edge also contribute to the decrease of activation energy with increasing impurity concentrations. Even though the activation energy is almost zero, the Te atoms are not necessarily all ionized.

\section{ACKNOWLEDGMENTS}

The authors wish to acknowledge Fred H. Eisen and Bryant M. Welch of Rockwell International Science Center for providing the Te-implanted samples. They also thank B. L. Crowder of IBM, Thomas J. Watson Research Center for doing variable energy $P$ implants.

\section{APPENDIX}

Dealing with the variation of activation energy with impurity concentrations, we included three effects in our model. These three effects are (i) the lowering of the conduction band edge due to attraction between conduction electrons and ionized donors, (ii) the broadening of the donor level due to donor-electron wave-function overlapping, and (iii) the smearing of the conduction band edge and the spreading of donor states due to potential fluctuation.

To obtain the lowering of conduction band edge, we proceeded as follows. Due to the overlapping of ionized donor potentials, the average barrier height against electron conduction has been reduced by an amount $\Delta E_{1}$,

$$
\Delta E_{1}=\frac{2 q^{2} \exp (d / 2 \lambda)}{4 \pi \epsilon \epsilon_{0}\left(\frac{1}{2} d\right)}-\frac{q^{2} \exp (-d / \lambda)}{4 \pi \epsilon \epsilon_{0} d},
$$

where $q$ is electronic charge, $\epsilon=11.8$ is the Si dielectric constant, $d$ is the average distance between ionized donors, and $\lambda$ is the screening length. The average ionized donor distance $d$ is given by

$$
d=\left(N_{D}^{+}\right)^{-1 / 3} \text {, }
$$

where $N_{D}^{+}$is the ionized donor concentration. The screening length $\lambda$ can be expressed in terms of electronic screening through $\lambda_{e}$ and ionized impurity screening length $\lambda_{i}$ by 


$$
\lambda=\left(\lambda_{e}^{-2}+\lambda_{i}^{-2}\right)^{-1 / 2}
$$

with

$$
\lambda_{e}=\left(\frac{\epsilon \epsilon_{0} K_{B} T}{q^{2} n}\right)^{1 / 2} \text { for the nondegenerate case, }
$$

and

$$
\begin{aligned}
\lambda_{i}= & \left(\frac{\epsilon \epsilon_{0} K_{B} T / q^{2}}{\left(n+N_{A}\right)\left(1-n+N_{A} / N_{D}\right)}\right)^{1 / 2} \\
& +\Gamma\left(\frac{4}{3}\right)\left[\frac{4}{3} \pi\left(N_{A}+N_{D}\right)\right]^{-1 / 3}
\end{aligned}
$$

where $K_{B}$ is the Boltzmann constant, $T$ is the absolute temperature, $n$ is the conduction electron concentration, $N_{D}$ and $N_{A}$ are donor and acceptor concentrations, respectively, and $\Gamma$ is the standard gamma function. Due to electronic tunneling through the barriers between potential wells, the bottom of the conduction band is located below the top of the barrier. Consequently, the total lowering of the conduction band edge is

$$
\Delta E_{c}=\Delta E_{1}+\Delta E_{2}
$$

where $\Delta E_{1}$ is given by Eq. (A1) and $\Delta E_{2}$, due to the electron tunneling effect, is approximately equal to

$$
\Delta E_{2}=2 \hbar^{2} / m^{*} d^{2},
$$

where $m^{*}=0.33 m_{e}$ is the $\mathrm{Si}$ effective mass and $d$ is given by Eq. (A2).

The average broadening of donor energy level $B$ has been found to be

$$
B=2 \int J(R) 4 \pi N_{D} R^{2} \exp \left(-\frac{4}{3} \pi N_{D} R^{3}\right) d R,
$$

where $J(R)$ is the energy transfer integral and is given by

$$
J(R)=\frac{Z_{\text {et: } q^{2}}}{4 \pi \epsilon \epsilon_{0}}(1+\xi R) \exp (-\xi R),
$$

where $Z_{\text {eff }}$ is the effective charge of the donor nucleus and $1 / \xi$ is the average radius of the donor electron wave function. $Z_{\text {eff }}$ and $\xi$ have different values for different impurities in Si. For impurities such as P, As, or $\mathrm{Sb}$ in $\mathrm{Si}$, the hydrogen model is appropriate and, thus we have

$$
Z_{\text {ef } \mathrm{f}}=1,
$$

and

$$
\xi=\left(1 / a_{H}\right)\left(E_{D} / E_{0}\right)^{1 / 2},
$$

where $a_{H} \equiv 4 \pi \epsilon \epsilon_{0} \hbar^{2} / m * q^{2}$ is the effective Bohr radius, $E_{0}=q^{2} / 8 \pi \epsilon \epsilon_{0} a_{H}$ is the ground-state energy from the effective mass theory ${ }^{20}$ and $E_{D}$ is the absolute value of the donor ionization energy at the low donor concentration case. Impurities such as $\mathrm{S}$ or $\mathrm{Te}$ in $\mathrm{Si}$ have two more valence electrons and thus the helium model seems to be appropriate. ${ }^{18,19}$ Using the helium model, we treated $Z_{\text {eff }}$ as a parameter such that

$$
E_{D}=E_{0}\left(Z_{\text {eff }}^{2}-\frac{5}{4} Z_{\text {eff }}+\frac{25}{128}\right),
$$

i.e.,

$$
Z_{\text {eff }}=\frac{5}{8}+\left(\frac{25}{128}+E_{D} / E_{0}\right)^{1 / 2},
$$

and we have

$$
\xi=\left(Z_{\text {eff }}-\frac{5}{16}\right) / a_{H} \text {. }
$$

Assuming constant density of states over the bandwidth $B$, we have the donor-level density of states,

$$
\begin{aligned}
\rho_{0}(E) & =N_{D} / B, & & -\frac{1}{2} B \leqslant E \leqslant \frac{1}{2} B \\
& =0 . & & \text { otherwise. }
\end{aligned}
$$

Due to the potential fluctuation, the conduction-band and donor-level density of states have been modified. The work of $\mathrm{Kane}^{21}$ and Morgan ${ }^{22}$ indicated that the potential fluctuation can be approximated by Gaussian distribution with the standard deviation given by

$$
\sigma=\left(\frac{\left(N_{D}^{+}+N_{A}\right) q^{4}}{8 \pi \epsilon^{2} \epsilon_{0}^{2}} \lambda\right)^{1 / 2}
$$

where $\lambda$ is the screening length, given by Eq. (A3). Thus, the potential fluctuation is given by

$$
p(V)=\left[1 /(2 \pi)^{1 / 2} \sigma\right] \exp \left(-V^{2} / 2 \sigma^{2}\right) .
$$

The conduction electron mobility is a function of energy in the conduction-band tail. For simplicity, we assume that the mobility is constant for electrons with potential energy $\geqslant-2 \sigma$ and mobility is zero for potential energy $<-2 \sigma$. The electrons with zero mobility would not contribute to current conduction. Therefore, the conduction-band density of states is given by

$$
\begin{aligned}
N_{c}(E) & =\frac{6 \sqrt{2}\left(m^{*}\right)^{3 / 2}}{m^{2} \hbar^{2}} \int_{-\infty}^{E}(E-V)^{1 / 2} p(V) d V, & & E \geqslant-2 \sigma \\
& =0, & & E<-2 \sigma .
\end{aligned}
$$

The donor-level density of states $\rho_{i}(E)$ should include both the fluctuation-induced broadening and the broadening due to the energy transfer integral. These two effects can be combined by averaging $\rho_{0}(E)$ given in Eq. (A15) over the value of the local potential. That is,

$$
\rho_{i}(E)=\int_{-\infty}^{\infty} \rho_{0}(E-V) p(V) d V,
$$

where $p(V)$ is given by Eq. (A17).

*Work supported in part by Air Force Cambridge Research Labs. (D.E. Davies).

${ }^{\dagger}$ Present address: Hewlett-Packard Laboratories, Palo Alto, Calif. 94304.

₹Present address: Intel Corporation, 3065 Bowers Avenue, Santa Clara, Calif. 95051 .

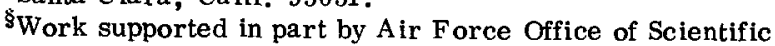

Research.

${ }^{1} \mathrm{~J}$. W. Mayer, L. Eriksson, and J.A. Davies, Ion Implantation in Semiconductors (Academic, New York, 1970).

${ }^{2}$ J. Gyulai, O. Meyer, R.D. Pashley, and J.W. Mayer, Radiat. Eff. 7, 17 (1971).

${ }^{3}$ S. T. Picraux, N. G.E. Johansson, and J.W. Mayer, Semiconductor Silicon (Electrochemical Society, Inc., New York, 1969), p. 422 .

${ }^{4} \mathrm{~S}$. Fischler, Metallurgy of Advanced Electronic Materials (Interscience, New York, 1963), Vol. 19.

${ }^{5}$ R. D. Pashley, Ph. D. thesis (California Institute of Technology, 1973 (unpublished).

${ }^{6}$ N. G. E. Johansson, J.W. Mayer, and O. J. Marsh, SolidState Electron. 13, 317 (1970).

${ }^{7} \mathrm{E} . \mathrm{H}$. Putley, The Hall Effect and Semiconductor Physics (Dover, New York, 1960). 
${ }^{8}$ R. Baron, G.A. Shifrin, O.J. Marsh, and J.W. Mayer, J. Appl. Phys. 40, 3702 (1969).

${ }^{9}$ R. L. Petritz, Phys. Rev. 110, 1254 (1958).

10 R. D. Pashley, Radiat. Eff. 11, 1 (1971).

${ }^{11}$ R. O. Schwenker, E.S. Pan, and R.F. Lever, J. Appl, Phys. 42, 3195 (1971).

${ }^{12}$ T.F. Lee and T.C. McGill, J. Appl. Phys. (to be published). ${ }^{13}$ W. J. Johnson and F. J. Gibbons, Projected Range Statistics in Semiconductors (Stanford University Bookstore, Stanford, Calif., 1969).

${ }^{14}$ L. J. van der Pauw, Phillips Res. Rep. 13, 1 (1958).

${ }^{15}$ N.G. E. Johansson and J.W. Mayer, Solid-State Electron.
$13,123(1970)$

${ }^{16}$ V.I. Fistul, Heavily Doped Semiconductors (Plenum, New York, 1969).

${ }^{17}$ G. L. Pearson and J. Bardeen, Phys. Rev, 75, 865 (1949).

${ }^{18}$ A. G. Milnes, Deep Level Impurities in Semiconductors (Interscience, New York, 1973).

${ }^{13}$ T.H. Ning and C.T. Sah, Phys. Rev. B 4, 3482 (1971). ${ }^{20}$ W. Kohn, Solid State Physics (Academic, New York, 1957), Vol. 5.

${ }^{21} \mathrm{E}$. O. Kane, Phys. Rev. 131, 79 (1963).

${ }^{22}$ T. N. Morgan, Phys. Rev. 139, A 343 (1965). 